\title{
Experimental Studies on the Motion and Discharge Behavior of Free Conducting Wire Particle in DC GIL
}

\author{
Jian Wang ${ }^{\dagger}$, Zhiyuan Wang*, Xiaoru Ni* and Sihua Liu*
}

\begin{abstract}
This study aims to restrain free conducting wire-type particles which are commonly and dangerously existing within DC gas-insulated transmission lines. A realistic platform of a coaxial cylindrical electrode was established by using a high-speed camera and a partial discharge (PD) monitor to observe the motion, PD, and breakdown of these particles. The probabilities of standing or bouncing, which can be affected by the length of the particles, were also quantitatively examined. The corona images of the particles were recorded, and particle-triggered PD signals were monitored and extracted. Breakdown images were also obtained. The air-gap breakdown with the particles was subjected to mechanism analysis on the basis of stream theory. Results reveal that the lifting voltage of the wire particles is almost irrelevant to their length but is proportional to the square root of their radius. Short particles correspond to high bouncing probability. The intensity and frequency of PD and the micro-discharge gap increase as the length of the particles increases. The breakdown voltage decreases as the length of the particles decreases.
\end{abstract}

Keywords: DC GIL, Free conducting wire particle, Motion patterns, PD features, Micro-discharge gap

\section{Introduction}

High reliability and transmission capacity have resulted in the wide use of gas insulation in electric power transmission and distribution components, such as gasinsulated switchgears (GIS) and gas-insulated transmission lines (GIL) [1]. With increasing demands for long-distance energy deliveries and large-scale offshore wind farms, direct current (DC) gas insulation apparatuses have been extensively investigated $[2,3]$. However, free-conducting particles can severely reduce insulation strength. In several cases, the rigidity of gases is as low as $10 \%$ of the uncontaminated value [4-7]. The insulation destruction of DC GIS/GIL caused by contaminated metal particles should be addressed more carefully than that of alternating current GIS/GIL because of an increased motion activity of such particles [8-10].

In a GIL system, metal particles are inevitably produced as a result of mechanical collisions, equipment vibration, or thermal expansion during GIL production, transportation, and assembly [11]. The movement and discharge of free particles are two principal mechanisms to decrease the insulating performance of GIL. Studies on these two mechanisms can be proposed as a theoretical basis for

$\doteqdot$ Corresponding Author: State Key Lab of Alternate Electrical Power System with Renewable Energy Sources, North China Electric Power University, Beijing, China. (1132101042@ncepu.edu.cn)

* State Key Lab of Alternate Electrical Power System with Renewable Energy Sources, North China Electric Power University, Beijing, China. (\{wangzhiyuan1002,15201667852\}@126.com, nixiaoru12 (a)163.com)

Received: September 26, 2016; Accepted: December 3, 2016 particle suppression measures, including setting particulate traps and using coatings or adhesive materials [12]. Therefore, the motion behavior and discharge mechanism of metal particles should be investigated theoretically and experimentally.

Spherical particles have been used as research subjects to analyze and calculate essential factors quantitatively and conveniently. But in practical projects, wire particles are more common and dangerous [13]. As such, conducting wire particles should be examined in detail. Qi et al. placed wire particles on an actual insulator in GIS and applied the Ultra High Frequency (UHF) method to capture the partial discharge signal triggered by metal particles. They found that the discharge process can be categorized into three stages, and the characteristic of partial discharge can be considered to determine the dangerous degree of particles [14]. Sakai et al. experimentally investigated the motion behavior and corona discharge caused by wire particles by using a simplified wedge-plate electrode system and observed that wire particles move randomly without specific patterns. Particles move to a high-electricfield region and then cause an air-gap breakdown [15].

Studies have focused on wedge-plate electrode systems and experiments have been conducted using metal particles attached to insulator surfaces. But studies on the motion behavior and discharge of free wire particles inside a coaxial cylindrical electrode system have been rarely performed. The characteristics and development of freeparticle-induced partial discharge and breakdown inside facilities are closely related to their motion behavior. However, these factors have yet to be further investigated. 
On the basis of the previous achievements, we established a realistic platform with a closed coaxial cylindrical electrode system comprising a high-speed camera to observe particle motion. We then experimentally and theoretically analyzed discharge and motion behavior. We also examined the characteristics of motion behavior by comparing the voltages of the particles used in the experiments with the numerical values derived from the following equations. We evaluated the probability effect caused by the length of particles on two motion patterns, including standing and bouncing and photographically obtained the breakdown in insulating gas. We further performed a mechanical analysis of the air-gap breakdown with free conducting wire particles on the basis of stream theory.

\section{Experimental Platform and Test Method}

A closed coaxial cylindrical electrode platform is established to observe the motion behavior and discharge caused by free particles as shown in Fig. 1. The DC voltage is generated by a signal generator (Agilent 33522A), then amplified 5000 times by a high voltage amplifier (Trek Model 50/12A) in series with a protective resistance. Finally, this voltage is imposed on the high voltage electrode. The shell of the specific experimental model shown in Fig. 2 is connected to the ground.

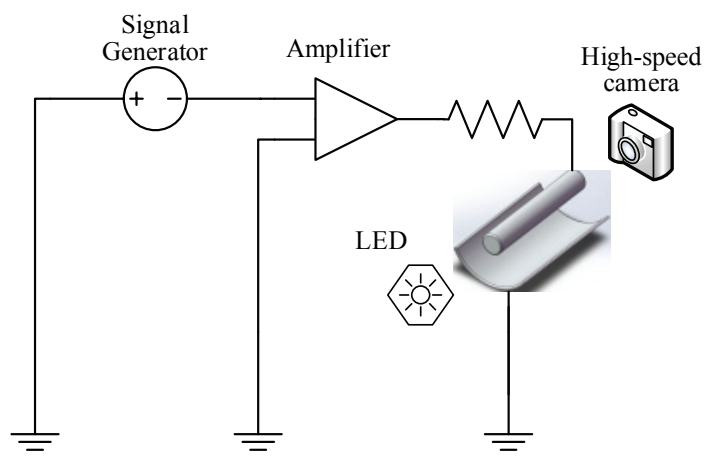

Fig. 1. Platform for the observation of particle movement and discharge

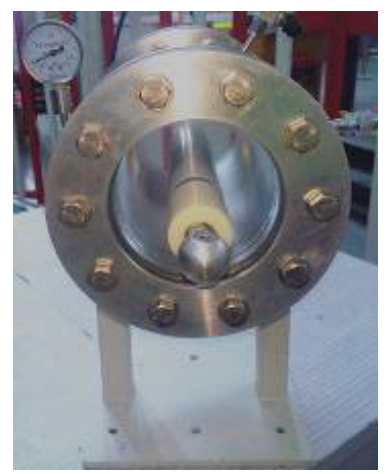

Fig. 2. Coaxial cylindrical experimental cavity
The conductors in the experimental cavity are all made of aluminum alloy. The outer radius of the high voltage conductor and the inner radius of the cavity shell are $R_{l}=20$ $\mathrm{mm}$ and $R_{2}=60 \mathrm{~mm}$, respectively. Three valves are supplied for gas in and out, namely, vacuum, $\mathrm{SF}_{6}$ charging, and $\mathrm{SF}_{6}$ recovery valves. Moreover, the conductor is equipped with a pressure gauge. Two circular plates made of silica glass are used for sealing off the ends of the shell. These plates are transparent enough to reinforce light from the outside and for a high-speed camera to observe the interior.

A high-speed camera (Fastec Hispec5) is utilized while reinforcing light using light-emitting diode (LED) to observe the motion behavior of particles. The light from LED can be adjusted coordinately when the Fastec Hispec5 is used to observe the gas breakdown path.

The wire particles adopted in this study are $0.1,0.2$, and $0.3 \mathrm{~mm}$ in radius. The lengths are $2,4,8,12$, and $18 \mathrm{~mm}$. The metal parts of cavity, such as the conductive rod, the shell, and particles, are all cleaned with ethyl alcohol to eliminate the effects of residual discharges on the initial voltage, motion behavior, and discharge characteristics of particles as shown in Fig. 2.

\section{Motion Behavior of Wire Particles}

The module units in DC GIL system are mainly straight in length of approximately 10-18 m. The non-uniform electric field in axial direction can be ignored in the areas that are far from the insulators and the interfaces of GIL. Then, the forces acting on the particles in DC GIL system were expressed as shown in Fig. 3, where the outer radius of high voltage conductor is $R_{I}$ on which the applied DC voltage is $U_{d c}$, whereas the inner radius of the shell is $R_{2}$. $R_{2}$ is connected to the ground. The particles are at the bottom of the shell as the initial position. Their radius is denoted as $a$, and the length is $l$. The following equation is derived to analyze the charging process and imposed force of particles.

The radius $a$ of particle is far less than the distance of $R_{2}-R_{1}\left(a \ll R_{2}-R_{1}\right)$. When the particle is at the bottom of

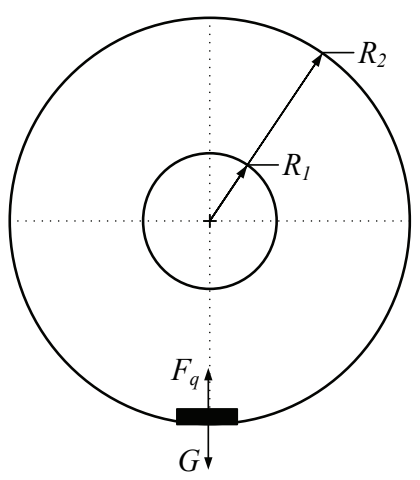

Fig. 3. Forces acting on the metal particle in coaxial cylindrical electrodes 
shell horizontally, the electric field around it can be derived as shown in Eq. (1):

$$
E_{R_{2}}=\frac{U_{d c}}{R_{2} \ln \frac{R_{2}}{R_{1}}} .
$$

Then, according to [16], the charge on the particle is positively proportional to its radius and length as shown in Eq. (2):

$$
q_{l}=2 \pi \varepsilon_{0} a L E=2 \pi \varepsilon_{0} a L \frac{U_{d c}}{R_{2} \ln \frac{R_{2}}{R_{1}}} .
$$

The Coulomb force $F_{q}$ under this condition is shown in Eq. (3):

$$
F_{q}=k E_{R_{2}} q_{l}=2 k \pi \varepsilon_{0} a L\left(\frac{U_{d c}}{R_{2} \ln \frac{R_{2}}{R_{1}}}\right)^{2}
$$

where $\varepsilon_{0}$ is the vacuum dielectric constant, $k$ is the correction factor derived from the mirror-image charge effect of surface charge, and $k=0.832$ [17] when the particle comes in contact with the electrodes.

The gravity $G$ is

$$
G=\pi a^{2} L \rho_{A l} g
$$

When the voltage is increased to a certain value $U_{\text {lift }}, F_{q}$ will overcome $G$. Then, the particles will move to the top. $U_{\text {lift }}$ is defined as the voltage when the particle moves from the bottom of the shell. According to $F_{q}=G, U_{\text {lift }}$ can be deduced as Eq. (5):

$$
U_{\text {lift }}=\left(\frac{a \rho_{A 1} \mathrm{~g}}{1.664 \varepsilon_{0}}\right)^{1 / 2} R_{2} \ln \frac{R_{2}}{R_{1}} .
$$

Eq. (5) indicates that $U_{\text {lift }}$ is positively correlated to the radius of particle $a$, having no correlation with the length $l$. Studies have proved that the influence of the composition and pressure of insulating gas imposed on the lifting moment of particles is negligible [18]. Therefore, the gas pressure is set as $0.1 \mathrm{MPa}$. The particles are $0.1,0.2$, and $0.3 \mathrm{~mm}$ in radius. $U_{\text {lift }}$ is recorded in Fig. 4. In Fig. 4, the solid line is the theoretical value of the lifting voltage, which can be drawn from the Eq. (5). And the experimental results on the radius of $0.1 \mathrm{~mm}, 0,2 \mathrm{~mm}$, and $0.3 \mathrm{~mm}$ are represented by the solid dots and error bars. The result shows that $U_{\text {lift }}$ in the experiments is consistent with that of Eq. (5). Moreover, the result indicates that particles that are similar in radius and different in length are close in $U_{\text {liff }}$.

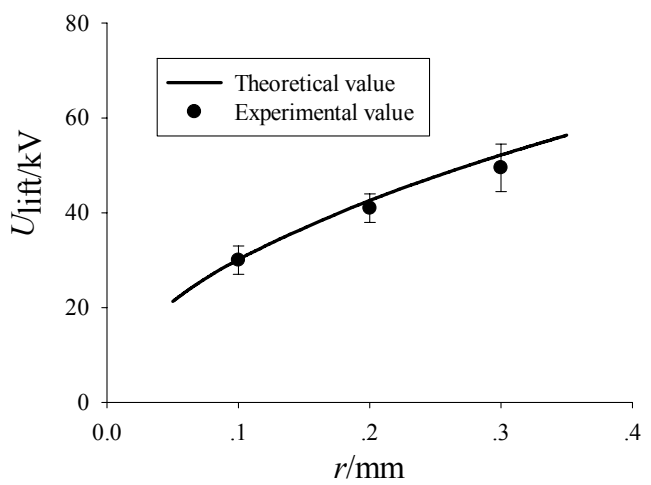

Fig. 4. Lifting voltages on different radii

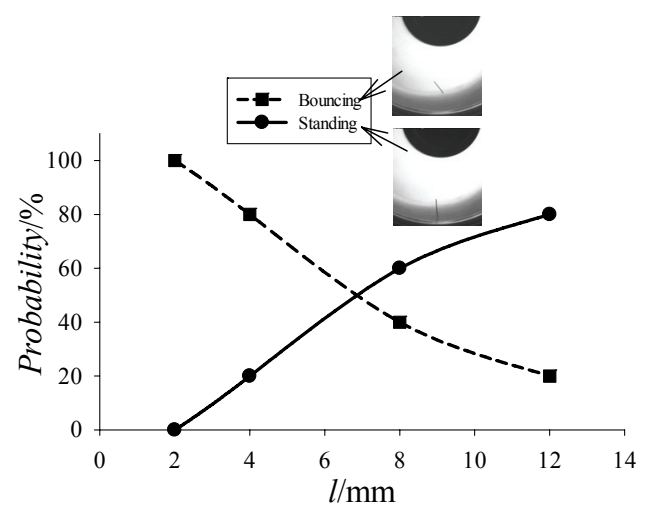

Fig. 5. Probabilities of motion patterns on different lengths

This finding proves that $U_{\text {lift }}$ is positively correlated to the radius $a$, but exhibits no correlation with the length $l$. The experimental result is consistent with the theoretical value.

The movement of particles is captured by a high-speed camera at $800 \mathrm{fps}$ with LED light. The movement shows that after particles moved upward, they will stand on the shell or bounce between high and ground electrodes as shown in Fig. 5. However, the probability to stand or to bounce relies on the length. For particles with same radius, the shorter the length of particles, the more likely for them to bounce. The longer the particles, the more likely for them to stand. The length surveyed is $2,4,8$ and $12 \mathrm{~mm}$ at a radius of $0.1 \mathrm{~mm}$. The statistical result is shown in Fig. 5. Fig. 5 shows the probabilities of motion patterns on different lengths. The experimental results on the length of $2 \mathrm{~mm}, 4 \mathrm{~mm}, 8 \mathrm{~mm}$ and $12 \mathrm{~mm}$ are represented by the solid dots as shown in Fig. 5. And the solid line and the dotted line are the fitting data of standing and bouncing probabilities respectively.

When $F_{q}$ surpasses $G$ slightly to lift the particle to suspend, corona discharge emerges at the tip of floating particle. The randomness that corona discharge takes on results in the randomness of the amount and distribution of the charges on particles. Thus, the randomness will finally cause the rotating torque that will pull up one particle tip first. Moreover, the randomness of the charge distribution will be reinforced if the gradient distribution of the electric 


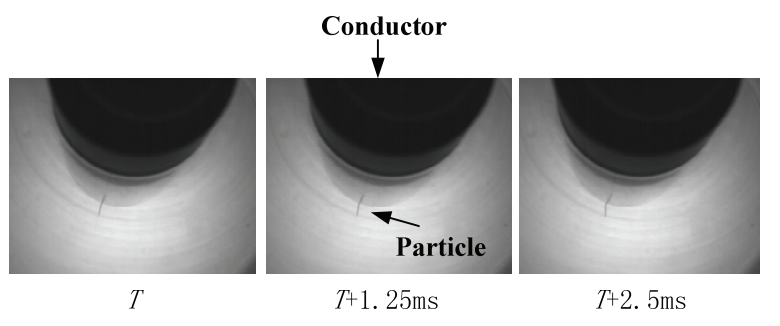

Fig. 6. Particle's motion of standing and spinning near the conductor under negative DC voltage

field in the coaxial cylindrical electrode system is considered. Consequently, for the particle that can lift when the voltage reaches $U_{\text {lift }}$, it will spin and jump up if its charge is sufficient. However, if its charge is not enough for $F_{q}$ to surpass $G$, it will not jump but stand on the ground electrode. Moreover, the particle will rotate slightly as a result of corona discharge. For particles with increased length, its $G$ is also increased and rotating torque is much less although the gradient force may be large. Thus, these particles are not likely to jump up but only stay standing. The standing particles will jump up and bounce between the electrode systems if the voltage is boosted further.

In addition to the influence of particle radius and length, the obvious effects of the voltage polarities on particle movement are found in the experiments. The probability of standing phenomenon on the shell is increased when the positive voltage is applied to the conductor. To analyze the reason, the particle near the negative-polarity shell starts to move and stand when the positive voltage is applied to the conductor. The discharge happens between the standing point of the particle and the shell. Electric field shielding is formed at the top. This result weakens the frontier field. The particles cannot easily jump, and standing phenomenon occurred often. Similarly, the particle near the positive-polarity shell starts to move and jump when negative voltage is applied to the conductor. The particles also stand on the conductor against the gravity. The particles stand and rotate on the conductor as shown in Fig. 6.

\section{Corona and PD}

Capturing the corona phenomenon is difficult when the particle jumps up. Thus, the particle at $18 \mathrm{~mm}$ length and $0.1 \mathrm{~mm}$ radius is used because it can remain standing according to Fig. 5. Negative DC voltage is applied in $0.1 \mathrm{MPa} \mathrm{SF}_{6}$ gas. The single-lens reflex cameras (Canon $5 \mathrm{~d}$ Mark II, $20 \mathrm{~s}$ exposure duration) is used to record the particle corona phenomenon in a completely dark environment.

As shown in Fig. 7, corona is observed on the particle's standing point. The intensity of the corona triggered by the micro-discharge increases with the voltage. Meanwhile, corona is also observed at the top of the particle. The



Fig. 7. Corona triggered by the $18 \mathrm{~mm}$-long wire particle under different voltages

corona increases and shows obvious polarity effect. When positive voltage is applied, the corona at the top of the particle is weak and presents a point of light. The corona at the top of the particle becomes intense and shows a brush discharge when the same level of negative voltage is applied. The model of the particle and the cavity are similar to the model of needle and plate electrode. Particle is negative when positive voltage is applied. When the voltage exceeds the corona's onset, the strong electric field at the top of the particles causes intense ionization producing a large number of electron-avalanche ionization. The diffusion distribution of space charge forms the plasma layer near the tip of particle, and plays the role of increasing the radius of curvature. The electric field between the top of particle and the conductor is weakened. Particle's corona shows a point of light. Particles become positive when negative voltage is applied. Streamer is formed at the top because of the different directions from the electric field. The head of streamer exhibits a positive space charge. This condition weakens the electric field in the plasma and strengthens the electric field of the head. This effect results in new electron-avalanche ionization at the position of the head. The electrons of streamer fly into the positive space charge region of the head strengthening and extending the discharge channel. Then, the high voltage area becomes forward. Therefore, the corona is intense when negative voltage is applied. The brush discharge phenomenon occurs.

The experiments are carried out in a SF6 gas of $0.1 \mathrm{MPa}$ using the Roche coil to monitor and record the leakage current in cavity using a particle radius of $0.1 \mathrm{~mm}$ and length of $2,4,8$, and $12 \mathrm{~mm}$. The partial discharge signals are extracted and analyzed. Motion states are also various because of the randomness of the motion of particles. Up to 200 screen data in each experiment are saved. The average discharge amplitude and average discharge time are counted.

Statistical results are shown in Figs. 8 and 9. Average discharge amplitude increases with the particle length, 


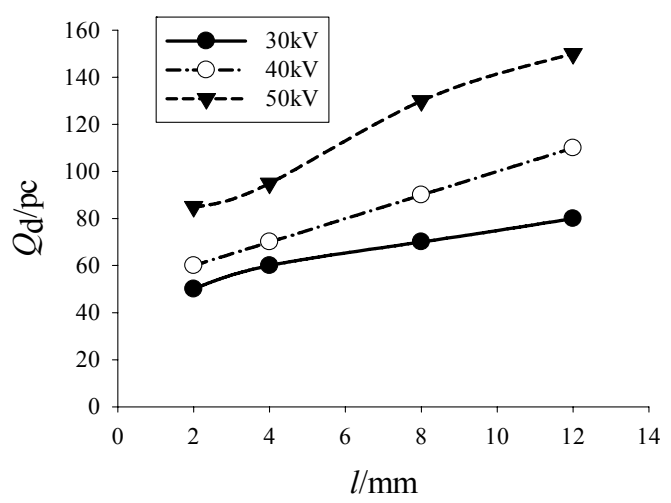

Fig. 8. Relationship between the mean discharge amplitudes and the length under different voltages

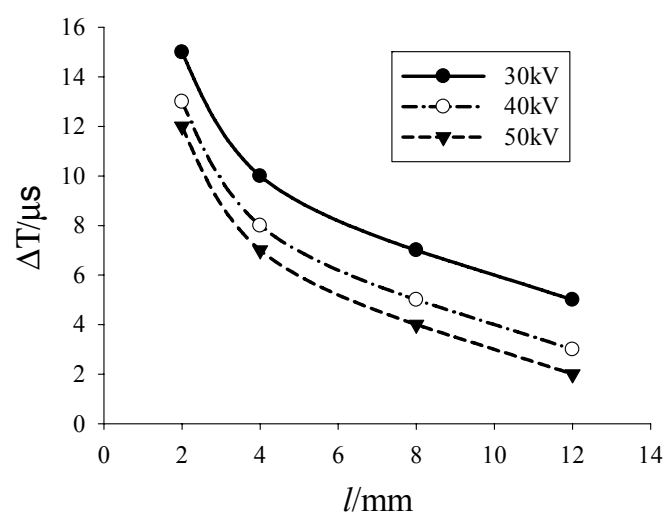

Fig. 9. Relationship between the mean discharge interval and the length under different voltages

whereas the average discharge time decreases with the particle length. This result suggests that at the same radius, the longer the particle, the more serious the partial discharge. The discharge amplitude increased by $60 \%-80 \%$ from $2 \mathrm{~mm}$ to $12 \mathrm{~mm}$. The discharge frequency increases up to five times. The higher the voltage, the higher the electric field intensity. Thus, the amplitude and frequency of the discharge both increased.

\section{Characteristic of Air-Gap Breakdown Caused by Free Wire Particles}

The breakdown characteristic caused by free particles is explored under normal air ignoring the influence of $\mathrm{SF}_{6}$ to determine the correlation between the air-gap breakdown and the motion behavior of particles. Particles of $2,4,8$, and $12 \mathrm{~mm}$ in length and $0.1 \mathrm{~mm}$ in radius are adopted. The voltage is boosted continuously until air-gap breakdown occurs. A high-speed camera at 10,000 fps accompanied with the weak light from LED is employed to facilitate the observation of the breakdown pattern.

Experiments are carried out to capture the images at the time $T$ when free particles jump upwards triggering the air-

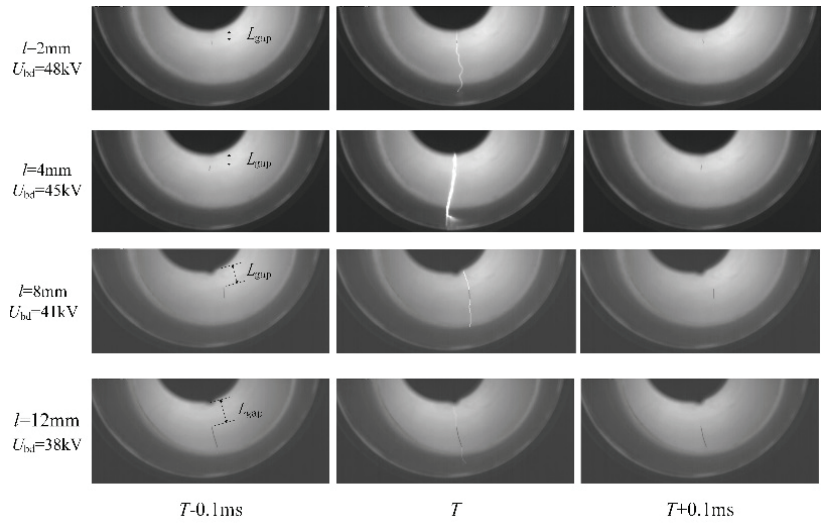

Fig. 10. Gas discharge triggered by the particles of different lengths

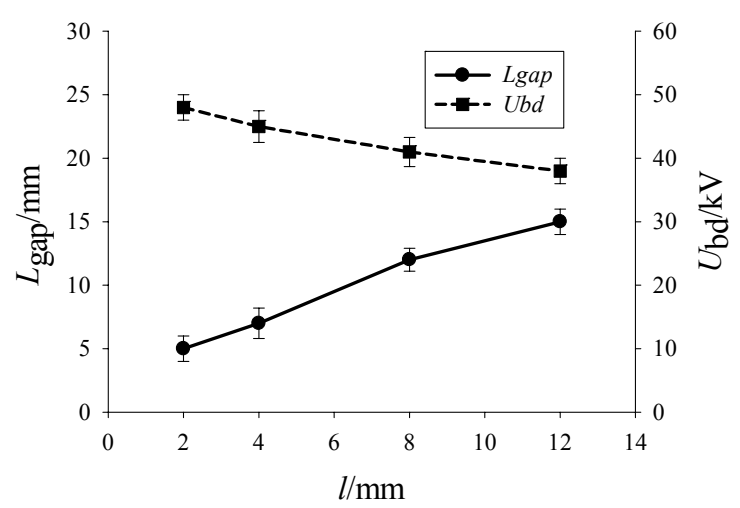

Fig. 11. Gap and voltage of breakdowns triggered by the particles at different lengths

gap breakdown. Pictures are shown in Fig. 10 at the time before, ongoing, and after the breakdown, namely, $T-0.1$ $\mathrm{ms}, T$, and $T+0.1 \mathrm{~ms}$, respectively.

In Fig. 10, a metal particle of $12 \mathrm{~mm}$ length causes $20 \%$ lower breakdown voltage than a metal particle of 2 $\mathrm{mm}$ length. During wire particle's jumping flight, the gap between the conductor and the shell will breakdown. Moreover, plasma channel is formed because of the effect of electric field distortion and the effect of short circuit along its length. The particles are always located in this conductive channel. The breakdown voltage $U_{\mathrm{b}}$ and microdischarge spacing $L_{\text {gap }}$ are recorded.

Fig. 11 indicates that the air-gap breakdown will be triggered along with a discharge channel in the direction of particle because of the distortion effect on the electric field and the short circuit effect along the particle length. The voltage at this moment and the gap distance between the particle and high voltage electrode are recorded as shown in Fig. 10.

The length of gap increases along with the length of particle while the breakdown voltage decreases. The reasons are drawn that the longer the length of particle is, the more charge it carries. Thus, the particle can trigger micro-discharge at a distance further from the high voltage 
electrode. Besides, the longer the particle, the larger the electric field along the gap; the self-sustained discharge in uniform field is likely to form [19]. The integration of electric field should reach the coefficient of electrons at the electron avalanche head to achieve the self-sustained discharge. This phenomenon results in long particle having long breakdown gap. By contrast, the particle will be seen contacting with the electrode forming a short circuit after the micro-discharge between the high voltage electrode and wire particle is triggered. Thus, the effect of microdischarge will be equivalent to that of a spike sticking out from the electrode. The virtual spike increases further because of the long gap reducing its distance from the outer shell. Therefore, for particles that can jump and bounce with the same radius, the increased length means decreased breakdown voltage and serious damage to the insulation system.

\section{Conclusion}

The lifting voltage is positively correlated with the radius of particle $a$ but is not correlated with length $l$. The slimmer the particle is, the more likely it is to be lifted and thus be more vivacious. Short particles likely bounce between electrodes, whereas long particles tend to remain standing after they are lifted from the bottom.

There exists corona discharge at the bottom of the standing particles. In the meantime, the corona on the top of the particle presents different states under different polarities of the voltage. The corona at the top of the particle is weak and presents a point of light when the positive voltage is applied. The corona at the top of the particle becomes intense and shows a brush discharge when the same level of negative voltage is applied. Particles cause partial discharge. At the same radius, the longer the particle, the more serious the partial discharge. The discharge amplitude increased by $60 \%-80 \%$ from 2 $\mathrm{mm}$ to $12 \mathrm{~mm}$. The discharge frequency increases up to five times. The higher the voltage, the higher the electric field intensity. Thus, the amplitude and frequency of the discharge both increased.

In bouncing, the electric field distortion triggers the airgap breakdown, and a short-circuit effect is caused by free wire particles. Moreover, the increased length of wire particles leads to an increased length of breakdown gap and a decreased breakdown voltage. Therefore, long and slim free particles possibly cause great danger to DC GIL insulation systems. This result should be further investigated to develop suppression measures.

\section{Acknowledgements}

The proposed research is supported by National Basic Research Program 973 of China (2014CB239502).

\section{References}

[1] H. Koch,V. Minisandram and A. Ficheux, Gas Insulated Substations, John Wiley, Chichester, UK, 2014, p. 10-25.

[2] R. Sarathi, A. V. Giridhar, A. Maniand and K. Sethupathi, "Investigation of partial discharge activity of conducting particles in liquid nitrogen under DC voltages using UHF technique," IEEE Trans. Dielectrics and Electrical Insulation, vol. 15, no. 1, pp. 655-662, Jun. 2008.

[3] Masanori Hara, Junya Suehiro, Hajime Maeda, Hideyuki Nakashima, "DC pre-breakdown phenomena and breakdown characteristics in the presence of conducting particles in liquid nitrogen," IEEE Trans. Dielectrics and Electrical Insulation, vol. 9, no. 1, pp. 23-30, Feb. 2002.

[4] J. R. Laghari and A.H. Qureshi, "A review of particlecontaminated gas breakdown," IEEE Trans. Electrical Insulation, vol. EI-16, no. 5, pp. 388-398, Oct. 1981.

[5] Alan H. Cookson, Owen Farish and George M. L. Sommerman, "Effect of conducting particles on ac corona and breakdown in compressed $\mathrm{SF}_{6}$," IEEE Trans. Power Apparatus and Systems, vol. PAS-91, no. 4, pp. 1329-1338, Jul. 1972.

[6] Alan H. Cookson and Owen Farish, "Particle-initiated breakdown between coaxial electrodes in compressed $\mathrm{SF}_{6}$," IEEE Trans. Power Apparatus and Systems, vol. PAS-92, no. 3, pp. 871-876, Jun. 1973.

[7] M. M. Morcos, H. Anis and K. D. Srivastava, "Particle-initiated corona and breakdown in GITL systems," IEEE Trans. Dielectrics and Electrical Insulation, vol. 24, no. 4, pp. 561-571, Aug. 1989.

[8] Koh-ichi Sakai, Dan Labrado Abella, Yasin Khan, Junya Suehiro and Masanori Hara, "Theoretical and experimental studies for spherical free conducting particle behavior between non-parallel plane electrodes with ac voltages in air," IEEE Trans. Dielectrics and Electrical Insulation, vol. 10, no. 3, pp. 404-417, Jun. 2003.

[9] Yasin Khan, Koh-ichi Sakai, Eun-Kyung Lee, Junya Suehiro and Masanori Hara, "Motion behavior and deactivation method of free-conducting particle around spacer between diverging conducting plates under DC voltage in atmospheric air," IEEE Trans. Dielectrics and Electrical Insulation, vol. 10, no. 3, pp. 444-457, Jun. 2003.

[10] Bo Qi, Chengrong Li, Zhaoliang Xing and Zhen Wei, "Partial discharge initiated by free moving metallic particles on GIS insulator surface: severity diagnosis and assessment," IEEE Trans. Dielectrics and Electrical Insulation, vol. 21, no. 2, pp. 766-774, Apr. 2014.

[11] Alan H. Cookson, "Electrical breakdown for uniform fields in compressed gases," Proceedings of the Institution of Electrical Engineers, vol. 117, no. 1, pp. 269-280, Jan. 1970. 
[12] A. H. Prakash, K. D. Srivastava and M. M. Morcos, "Movement of particles in compressed $\mathrm{SF}_{6}$ GIS with dielectric coated enclosure," IEEE Trans. Dielectrics and Electrical Insulation, vol. 4, no. 3, pp. 344-347, Jul. 1997.

[13] R. M. Radwan, R. Morsi and M. A. Abd-Allah, "Motion of free conducting particles in $\mathrm{SF}_{6}$ insulated systems under dc switching voltages," IEEE Trans. Dielectrics and Electrical Insulation, vol. 1, no. 1, pp. 25-30, Feb. 1994.

[14] Bo Qi, Cheng Rong Li, Zhen Hao and Bi Bo Geng, "Surface discharge initiated by immo-bilized metallic particles attached to gas insulated substation insulators: process and features," IEEE Trans. Dielectrics and Electrical Insulation, vol. 18, no. 3, pp. 792-800, Jun. 2011.

[15] Koh-ichi Sakai, Shin-Ichiro Tsuru, Dan Labrado Abella and Masanori Hara, "Conducting particle motion and particle-initiated breakdown in dc electric field between diverging conducting plates in atmospheric air," IEEE Trans. Dielectrics and Electrical Insulation, vol. 6, no. 1, pp. 122-130, Feb. 1999.

[16] Jiangbo Jia, Fengbo Tao, Lanjun Yang and Qiaogen Zhang, "Motion analysis of spherical free conducting particle in non-uniform electric field of GIS under DC voltage," Proceedings of the CSEE, vol. 26, no. 8, pp. 106-111, Apr. 2006.

[17] N. N. Lebedev, I. P. Skalskaya, "Forces acting on a conducting sphere in field of a parallel plate condenser," Soviet Physics-Technical Physics, vol. 7, no. 3, pp. 268-270, Mar. 1962.

[18] M. Morcos, S. Zhang, K. Srivastava and S. M. Gubanski, "Dynamics of metallic particle contaminants in GIS with dielectric-coated electrodes," IEEE Trans. Power Delivery, vol. 15, no. 2, pp. 455460, Apr. 2000.

[19] Kazutoshi Asano, Ryohei Hishinuma, Kyoko Yatsuzuka, "Bipolar DC corona discharge from a floating filamentary metal particle," IEEE Trans. Industry Applications, vol. 38, no. 1, pp. 57-63, Jan/ Feb. 2002.

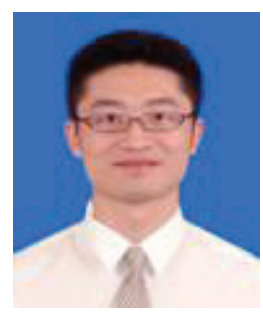

Jian Wang was born in Shandong, China in 1985. He received his M.Sc. degree with a major in electric power system, from Shandong University, China, in 2010. He is currently a Ph.D. student at the North China Electric Power University, China.

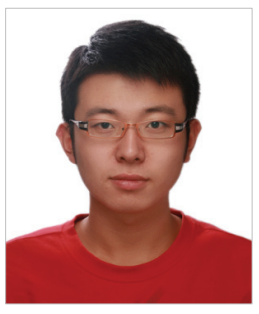

Zhiyuan Wang was born in Shandong, China in 1992. He received the B.S.E.E degree in electrical engineering and automation from North China Electric Power University, China, in 2015 . He is currently a postgraduate student at the North China Electric Power University, China.

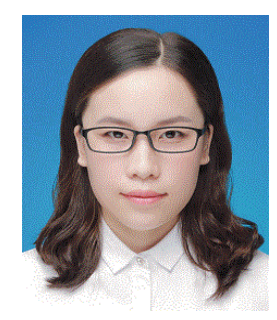

Xiaoru Ni was born in Shanxi, China in 1994. She will receive the B.S.E.E degree inelectrical engineering and automation from North China Electric Power University, China, in 2017. She is currently an undergraduate at the North China Electric Power University, China.

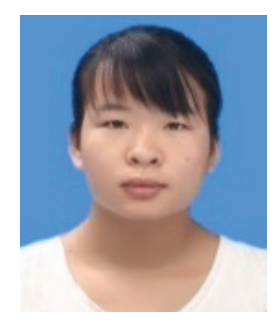

Sihua Liu was born in Shandong, China in 1992. She received the B.S.E.E degree in electrical engineering and automation from North China Electric Power University, China, in 2014. She is currently a postgraduate studentat the North China Electric Power University, China. 